\title{
Interleukin-12 as successful adjuvant in tuberculosis treatment
}

\author{
U. Greinert*, M. Ernst* ${ }^{\#}$ M. Schlaak*, P. Entzian*
}

\begin{abstract}
Interleukin-12 as successful adjuvant in tuberculosis treatment. U. Greinert, M. Ernst, M. Schlaak, P. Entzian. C ERS Journals Ltd 2001.

ABSTRACT: Interleukin-12 (IL-12) proved to be an effective and successful adjuvant to a standard antituberculotic medication in a patient suffering from progressive clinical tuberculosis (TB).

IL-12 is a potent enhancer of interferon- $\gamma$ production which is necessary for killing intracellular bacteria like mycobacteria. This patient's TB was progressive, although sensitivity to first-line antituberculotics was proven and medication was given as directly observed therapy over more than 8 months. The 3-month adjuvant therapy with IL-12 significantly and convincingly improved results.
\end{abstract}

It is believed that this case, the first in the literature to describe adjuvant interleukin12 therapy in tuberculosis, strongly encourages the study of adjuvant interleukin-12 therapy on a more systematic basis.

Eur Respir J 2001; 17: 1049-1051.

\author{
*Dept Internal Medicine, and ${ }^{*}$ Dept \\ Immunology and Cell Biology, Research \\ Centre Borstel, Borstel, Germany
}

Correspondence: U. Greinert, Dept Internal Medicine, Research Centre Borstel, Parkallee 35, D-23845 Borstel, Germany.

Fax: 494537188313

Keywords: Cytokines interleukin-12

interferon- $\gamma$

tuberculosis

Received: May 292000

Accepted after revision October 13 2000
The presence of interferon- $\gamma(\mathrm{IFN}-\gamma)$ at the site of infected phagocytes is a prerequisite for killing intracellular bacteria such as mycobacteria [1]. Defective IFN- $\gamma$ production of peripheral blood mononuclear cells (PBMC) in patients with tuberculosis (TB) has been demonstrated [2], and SoDHI et al. [3] described close correlations between bolt radiographic extent of pulmonary TB and extrapulmonary disease in human immunodeficiency virus (HIV) positive and negative patients and low IFN- $\gamma$ production. The most potent enhancer of IFN- $\gamma$ release of preactivated T-cells is interleukin (IL)-12 [4]. From recent data it is known that IL-12 serum levels in patients with TB do not correspond to disease activity [5]. Analysis of bronchoalveolar lavage fluid showed an increase of cells expressing messenger ribonucleic acid (mRNA) for the $\beta 1$ and $\beta 2$ subunits of the IL-12 receptor in active pulmonary TB [6]. For other sites of tuberculous disease i.e. tuberculous pleurisy or lymphadenitis IL-12, receptor- $\beta 2$ mRNA expression was found to be increased in vivo resulting in elevated IFN- $\gamma$ production [7].

\section{Case report}

A 24-yr-old male $(167 \mathrm{~cm}, 50.1 \mathrm{~kg})$, born in Burma and residing in Germany since 1995, was hospitalized in April 1998 with suspected TB. The patient was seronegative for HIV 1/2 with no former history of TB. Pulmonary TB with miliary spread and TB involvement of cervical lymph nodes was diagnosed. Isolates of Mycobacterium tuberculosis were sensitive to all firstline anti-TB drugs. Combined therapy with isoniazid
(0.3 g), rilampicin (RMP; $0.45 \mathrm{~g}$ ), eltambutol (EMB; $1.0 \mathrm{~g}$ ) and pyrazinamide (PZA; $1.5 \mathrm{~g}$ ) was initiated. Medication was administered as directly observed oral therapy (DOT) and later intravenously in case of malabsorption of antituberculous drugs, when there was clinical progress of disease. Nevertheless, symptoms (fever, cough) progressed with intravenous treatment during the following weeks, and DOT by mouth was reinstituted. The patient developed cervical and mediastinal abscesses at various sites requiring repeated surgical incisions. $M$. tuberculosis found in pus from the abscesses showed an identical drug sensitivity pattern. The pulmonary lesions improved and the latest isolate of M. tuberculosis was cultured in October 1998, still showing sensitivity to the drugs used. Fever, however, persisted, further cervical abscesses occurred and splenic and abdominal lymph node involvement was suspected from computed tomography (CT) scans. The patient was transferred to Borstel in mid-October 1998 (for chest radiograph see fig. 1a). Here, DOT was continued, using isoniazid (INH), EMB, RMP and PZA until November 1998 when, due to increases in liver enzymes, RMP was replaced by rifabutin with the patient's $M$. tuberculosis strain being susceptible to that drug. PZA was replaced by streptomycin (SM) in December and a cumulative dose of $30 \mathrm{~g}$ was given. Lung involvement improved further, but a bone defect was detected in the skull, and new cervical (fig. 1b), abdominal and mediastinal abscesses developed with one of them in the immediate vicinity of the left pulmonary artery. A smear of pus from a newly developing forefoot lesion was positive for acid-fast bacilli and deoxyribonucleic acid (DNA) of the M. tuberculosis-complex was detected using the nucleic 

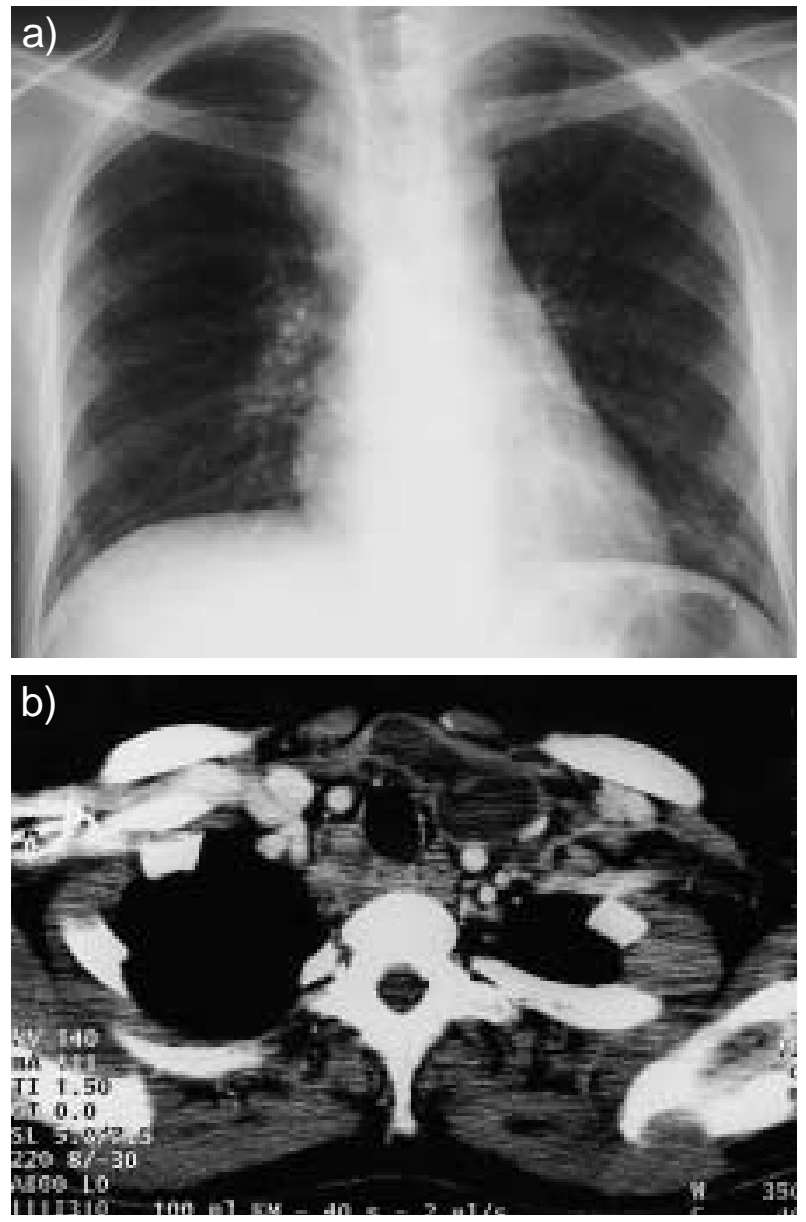

Fig. 1. - a) Chest radiograph in October 1998 showing paratracheal enlargement of the mediastinum to the right. b) Computed tomography scan of the neck showing cervical abscesses (December 1998).

acid amplification technique, but culture remained negative. There were no co-existing diseases and inadequate drug absorption was excluded by monitoring INH and RMP serum levels. Fluorescence-activated cell sorter (FACS) analyses of cell differentials in peripheral blood prior to adjuvant cytokine treatment showed raised counts of activated T-cells $(\mathrm{CD} 25+, \mathrm{CD} 38+)$. IFN- $\gamma$ release in in vitro stimulated cultures of diluted whole blood (DWB) and PBMC was, however, impaired significantly. The patient gave informed consent to cytokine treatment, and IFN- $\gamma$ was injected subcutaneously ( 1 daily injection of $1.5 \times 10^{6} \mathrm{U}$ IFN- $\gamma \mathrm{lb}$, Imukin ( , Boehringer Ingelheim, Germany) in addition to the above TB drugs. Ex vivo-in vitro stimulated IFN- $\gamma$ release was augmented in follow-up studies (see fig. 2), but the clinical course of the patient did not improve as expected during 4 months of treatment and new cervical abscesses appeared. IFN- $\gamma$ prescription was discontinued. As in vitro IFN- $\gamma$ cell production can be augmented by addition of IL-12 [8], whether the PBMC of the patient would show enhanced IFN- $\gamma$ response to stimulation in the presence of IL-12 in vitro was tested.

It was found that the addition of IL-12 restored the impaired IFN- $\gamma$ release of both antigenic stimulation by purified protein derivative (PPD) as well as the

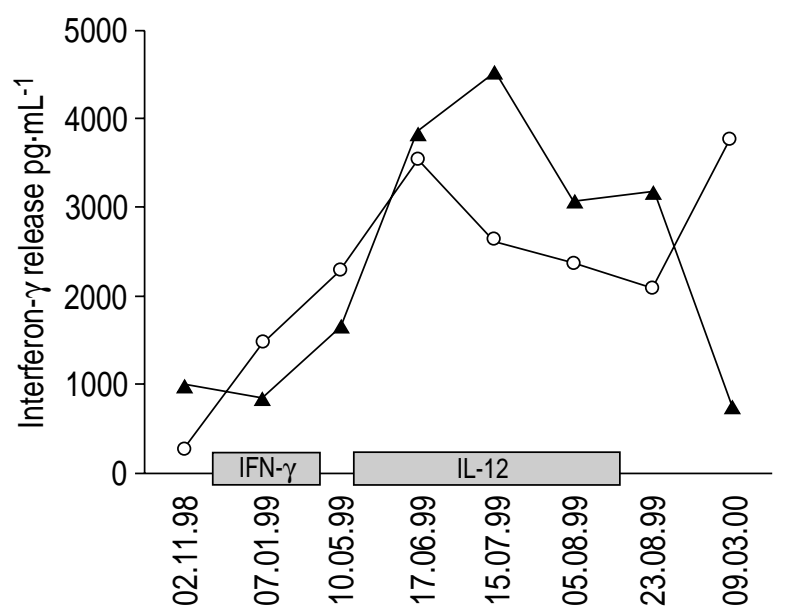

Fig. 2. - Interferon (IFN)- $\gamma$ concentrations in culture supernatants of patient cells. Cells were cultured as peripheral blood mononuclear cells, and were stimulated either with purified protein derivative $(\bigcirc)$ or anti-CD3 $(\boldsymbol{\Delta})$. Grey bars showing cytokine names mark times of adjuvant cytokine treatment. IL-12: interleukin-12.

polyclonal stimulation by phytohaemagglutination (PHA) and anti-CD3 monoclonal antibodies. In unstimulated PBMC, however, IL-12 did not induce IFN- $\gamma$ release (fig. 3). Thus it was concluded, that the impaired IFN- $\gamma$ release capacity of the patient's T-cells was not due to a major IL-12 receptor defect. Therefore, it was expected that treatment of the patient with IL-12 would enhance the IFN- $\gamma$ release from mycobacteria-specific memory T-helper cells at the sites of infection, and thus enhance the local antimycobacterial activities of both Tcells and monocytes/macrophages. Adjuvant treatment was started with this cytokine for 3 months $(300 \mathrm{ng} \cdot \mathrm{kg}$ body weight ${ }^{-1}$ subcutaneously twice weekly, recombinant IL-12, Genetics Institute, USA). Prior consent to IL-12 treatment was obtained from the patient and local ethics committee. While using additional cytokine therapy, triple drug therapy with INH rifabutin and

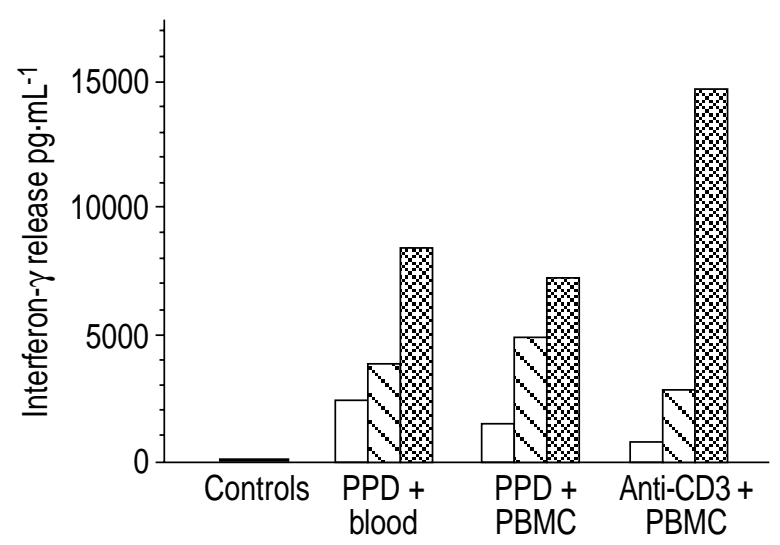

Fig. 3. - In vitro interferon- $\gamma$ release of diluted whole blood (blood) or peripheral blood mononuclear cells (PBMC) in January 1999. Cultures were stimulated by purified protein derivative (PPD) or anti-CD3 and incubated with or without addition of interleukin-12 in concentrations of $1 \mathrm{ng} \cdot \mathrm{mL}^{-1}$ or $10 \mathrm{ng} \cdot \mathrm{mL}^{-1}$. $\square$ : control; $\mathbb{\mathbb { N }}$ : incubated with $1 \mathrm{ng} \cdot \mathrm{mL}^{-1} ; \mathbf{r}: 10 \mathrm{ng} \cdot \mathrm{mL}^{-1}$. 
EMB was taken. IL-12 therapy initially led to normalization of PPD-IFN- $\gamma$ release of cultured PBMC and DWB (fig. 2), and, most importantly, improved the patient's clinical course significantly: no new abscesses occurred and the mediastinal abscesses diminished as did the skull bone abnormality. The chest radiogram normalized but the splenic abnormality persisted. The sole side-effects of cytokine therapy were fever subsequent to drug injection and slight asthenia.

On follow-up examination 6 months after cessation of IL-12 treatment, there were only minor changes in lymph node size at different sites, but a cervical lymph node of $2 \mathrm{~cm}$ in diameter with a draining sinus had developed. The patient continued to take triple drug therapy as previously discussed. Due to lack of availability of IL-12, IFN- $\gamma$ therapy was reinstituted for 4 weeks without any clinical benefit. Three weeks after reapplication of IL-12, healing of the cervical lesion was documented with reduction of lymph node size to $1 \mathrm{~cm}$. IL-12 therapy in combination with triple drug therapy was prescribed for a further 5 months.

\section{Discussion}

Presented is an unusual case of a patient with disseminated TB and a disparity between pulmonary and extrapulmonary responses to therapy. Although sensitivity to standard antituberculous drugs was proven, lung involvement improved while extrapulmonary manifestations deteriorated.

It was demonstrated by coincubation with different amounts of IL-12, an enhanced IFN- $\gamma$ release of PPD or anti-CD3-stimulated, but not of unstimulated PBMC in vitro, and only addition of IL-12 to the therapeutic regimen convincingly improved clinical and laboratory parameters in the patient. Systemic application of IFN- $\gamma$ was of no clinical benefit. The in vitro testing of the IFN- $\gamma$ release enhancing capacity of IL12 in the patient's PBMC was essential in the present trial since by this means inherited IL-12 receptor deficiency, which has been described in some cases of patients with impaired mycobacterial immunity [9], could be excluded. The data of INF- $\gamma$ release while the patient was being treated with IL-12 show that on August 5 in vitro stimulated IFN- $\gamma$ release was reduced compared to July 15 , when the same treatment regimen was applied, although concentrations were still significantly above those measured prior to cytokine treatment. In cultures of isolated PBMC it appears that this could be due in part to the relatively smaller proportion of non-B-lymphocytes within the PBMC on August 5 than on July 15, since the reduction in IFN- $\gamma$ release was annulled when related to equal amounts of non-B-cells.

With respect to the disparity between pulmonary and extrapulmonary improvement on antituberculous therapy, there is experimental data pointing to an association of splenomegaly with destruction of lymphoid tissue structure and increased apoptosis as a possible important component of ineffective immune response against mycobacterium-induced immunopathology [10]. This set of data is in accordance with the clinical observation of a case of disseminated TB unresponsive to chemotherapy until splenectomy was performed [11]. In the patient splenomegaly with persisting pathological changes of the spleen on CTscan was documented.

It is believed that IFN- $\gamma$ application was less effective than interleukin-12 due to insufficient cytokine concentrations at the sites of infection (the dose used was limited by side-effects, i.e. fever etc.). By contrast, addition of interleukin-12 was a better-tolerated stimulant of prestimulated cells. The results encourage the study of interleukin-12 effects in tuberculosis patients on a more systematic basis.

Acknowledgements. The authors wish to thank Genetics Institute, Munich, Germany for kindly providing the recombinant cytokine Interleukin-12 and patient insurance.

\section{References}

1. Murray HW. Interferon-gamma and host antimicrobial defence: current and future clinical applications. Am J Med 1994; 97: 459-467.

2. Vilcek J, Klion A, Henriksen-DeStefano D, et al. Defective gamma-Interferon production in peripheral blood leukocytes of patients with acute tuberculosis. $J$ Clin Immunol 1986; 6: 146-151.

3. Sodhi A, Gong J, Silva C, Quian D, Barnes PF. Clinical correlates of Interferon- $\gamma$ production in patients with tuberculosis. Clin Infect Dis 1997; 25: $617-620$.

4. Trinchieri G. Interleukin-12: a cytokine at the interface of inflammation and immunity. $A d v$ Immunol 1998; 70: 83-243.

5. Verbon A, Juffermans N, van Deventer SJH, van Deutekom H, Spealman P, van der Poll T. Serum concentrations of cytokines in patients with active tuberculosis and after treatment. Clin Exp Immunol 1999; 115: $110-113$

6. Taha RA, Minshall EM, Olivenstein R, et al. Increased expression of IL-12 receptor mRNA in active pulmonary tuberculosis and sarcoidosis. Am J Respir Crit Care Med 1999; 160: 1119-1123.

7. Zhang M, Gong J, Presky DH, Xue W, Barnes PF. Expression of the IL-12 receptor $\beta 1$ and $\beta 2$ subunits in human tuberculosis. J Immunol 1999; 162: 2441 - 2447.

8. McDyer JF, Hackley MN, Walsh TE, Cook JL, Seder RA. Patients with multidrug-resistant tuberculosis with low CD4 $+\mathrm{T}$ cell counts have impaired Th1 responses. J Immunol 1997; 158: 492 - 500.

9. Altare F, Durandy A, Lammas D, Biet F, Ameisen JC, Locht L. Impairment of mycobacterial immunity in human interleukin-12 receptor deficiency. Science 1998; 280: 1432 - 1435 .

10. Kremer L, Estaquier J, Wolowczuk I, Williams RR, Khanduri P. Ineffective cellular immune response associated with $\mathrm{T}$-cell apoptosis in susceptible Mycobacterium bovis BCG-infected mice. Infect Immun 2000; 68: 4264-4273.

11. Nayyar V, Ramakrishna B, Methew G, et al. Response to antituberculous chemotherapy after splenectomy. J Intern Med 1993; 233: 81-83. 